\title{
Herba Rhodiolae alleviates depression via the BDNF/TrkB-GSK-3 $\beta$ signaling pathway
}

\author{
Lili Gao, Chenghan Wu, Yuansheng Liao, Sheng Zhang, Jian Zhao \\ Department of Neurology and Psychiatry, The Second Affiliated Hospital of Fujian Traditional Chinese Medical University, Fuzhou, China \\ Contributions: (I) Conception and design: L Gao; (II) Administrative support: L Gao; (III) Provision of study materials or patients: C Wu; (IV) \\ Collection and assembly of data: Y Liao; (V) Data analysis and interpretation: S Zhang, J Zhao; (VI) Manuscript writing: All authors; (VII) Final \\ approval of manuscript: All authors. \\ Correspondence to: Lili Gao. Department of Neurology and Psychiatry, The Second Affiliated Hospital of Fujian Traditional Chinese Medical \\ University, 282 Wusi Road, GuLou District, Fuzhou 350003, China. Email: gaolilidexingfu@163.com.
}

\begin{abstract}
Background: Depression is one of the most common psychiatric disorders worldwide. Many antidepressant drugs are only effective for specific patients and their long-term use can lead to treatment resistance. There is an ongoing need for more effective antidepressant treatments.

Methods: The rats were exposed to chronic unpredictable mild stress (CUMS) to induce depression model. The glutamate level in the hippocampus was determined by high performance liquid chromatography (HPLC). Protein levels in the tissues were measured using western blotting. The fluid consumption test was performed to evaluate potential anhedonia. Open field test was conducted to evaluate locomotor activity. Forced swimming test was used to evaluate susceptibility to negative mood.

Results: In the present study, the depressive-like behaviors induced by CUMS were alleviated in rats by treatments with Herba Rhodiolae $(0.04 \mathrm{~g} / \mathrm{kg}, 1 \mathrm{~mL})$, venlafaxine $(0.035 \mathrm{~g} / \mathrm{kg}, 1 \mathrm{~mL})$, and combination of Herba Rhodiolae and glycogen synthase kinase 3 beta (GSK-3 $\beta)$ inhibitor $(10 \mu M, 1 \mathrm{~mL})$. Among these treatments, the combination of Herba Rhodiolae and GSK-3 $\beta$ inhibitor was most effective. Abnormalities in the glutamate system and autophagy of the brain were decreased greatly in Herba Rhodiolae and GSK$3 \beta$ inhibitor-treated CUMS rats. Herba Rhodiolae was more effective in promoting the brain derived neurotrophic factor/tropomyosin receptor kinase B (BDNF/TrkB) signaling. The expression of GSK-3 $\beta$ was remarkably suppressed by the GSK-3 $\beta$ inhibitor.
\end{abstract}

Conclusions: These findings indicated that the combined effects of Herba Rhodiolae and GSK-3 $\beta$ inhibitor contributed to the activation of BDNF/TrkB-GSK-3 $\beta$ signaling pathway.

Keywords: Herba Rhodiolae; depression; BDNF/TrkB-GSK-3 $\beta$ signaling pathway

Submitted Oct 13, 2021. Accepted for publication Dec 01, 2021.

doi: 10.21037/atm-21-5849

View this article at: https://dx.doi.org/10.21037/atm-21-5849

\section{Introduction}

Depression is one of the most common psychiatric disorders worldwide. The illness is clinically characterized by various symptoms including persistent sadness, loss of interest and enjoyment, feelings of guilt, anxiety, fatigue, low self-worth, poor concentration, and recurrent suicidal thoughts (1). Depression threatens the physical and psychological health of patients and greatly affects their work and personal lives, contributing to the increasing rate of suicide of this century
$(2,3)$. Previous findings have demonstrated a complex of factors contributing to the development of depression, including genetic, environmental stress, endocrine, and immunologic factors. Some molecular pathways are associated with the development of depression, including reductions of brain monoaminergic transmission, function of neurotransmitter receptors and neurotrophic factors, increase of proinflammatory cytokines, abnormality of nitric oxide, oxidative disturbances, and dysregulation of 
the hypothalamic-pituitary-adrenal axis (4). Nonetheless, the pathogenesis of depression has not been well clarified and effective antidepressant agents require further exploration. Physiological mechanisms of an anti-depressive effect of physical exercise in major depressive disorder has been believed to be closely linked with brain-derived neurotrophic factor (BDNF) (5-7), and BDNF/TrkB/GSK$3 \beta$ pathway has been proved to be closely related with the development of depression (5-7). GSK-3 $\beta$ is an enzyme that phosphorylates glycogen synthase, which in turn inhibits glycogen biosynthesis. Moreover, TrkB/GSK-3 $\beta$ is now believed to play an important role in the pathophysiology of depression and is implicated to be a drug target for the treatment of depression (7).

There are 3 types of treatment targeting depression, namely, electroconvulsive therapy, psychotherapy, and pharmacotherapy. Among them, electroconvulsive therapy is the oldest and most controversial treatment, given the cognitive side effects, and its application has dwindled despite it having been proven effective in some cases (8). The psychotherapeutic techniques are composed of cognitive behavioral therapy, interpersonal psychotherapy, problem-solving therapy, non-directive supportive therapy, behavioral activation therapy, and exercise. Psychological treatment is effective for moderate depression, but its efficacy appears to be limited for chronic and severe depression (9). Antidepressant medications have become increasingly prevalent in recent decades, and the drugs mainly include tricyclic antidepressants, monoamine oxidase inhibitors, selective serotonin reuptake inhibitors, and serotonin norepinephrine reuptake inhibitors (10). However, such drugs may only be effective for specific patients and long-term utilization can lead to treatment resistance (11). Besides, side effects and pathogenesis have been reported as associated with most pharmacotherapies (12).

Traditional Chinese medicine (TCM) has been increasingly applied to treat depression in China (13). Alongside the associated safety advantages, TCM herbal formulae could be more effective and less prone to treatment resistance, given its multiple targets, compared to conventional chemical drugs (14). Rhodiola rosea root, an herbal medicine known as "golden root" or "arctic root", has been used as an antidepressant for years, and clinical trials have shown that it is effective (15). Previous findings have indicated that Rhodiola rosea contains hundreds of bioactive compounds, for example, phenylpropanoids, phenylethanol derivatives, flavanoids, monoterpernes, triterpenes, and phenolic acids (16), which are supposed to be involved in neuroprotective, cardio-protective, antifatigue, anti-stress, anti-depressive, and anxiolytic activities, and immunity improvement (17). Nonetheless, the molecular mechanism of Rhodiola rosea in the treatment of depression is still unknown.

We present the following article in accordance with the ARRIVE reporting checklist (available at https://dx.doi. org/10.21037/atm-21-5849).

\section{Methods}

\section{Animals}

Adult male Sprague-Dawley (SD) rats (8 weeks, $220-240 \mathrm{~g}$ ) were purchased from Hangzhou Medical College, Hangzhou, China. The rats were separately housed in the animal facility under the $12 \mathrm{~h}$ light-dark cycle at $23-25^{\circ} \mathrm{C}$. The humidity was around $50 \%$, and the rats were provided free access to food and water. After 7 days of acclimatization, 30 rats were randomly divided into 5 groups. The rats in group I (Sham group) were treated orally with physiological saline ( $1 \mathrm{~mL}$ per day). Rats in all other groups were exposed to chronic unpredictable mild stress (CUMS), combined with physiological saline (Oral, $1 \mathrm{~mL}$ per day) in group II, Herba Rhodiolae suspension (Oral, $0.04 \mathrm{~g} / \mathrm{kg}$, once a day) in group III, Herba Rhodiolae (Oral, $0.04 \mathrm{~g} / \mathrm{kg}$, once a day) and glycogen synthase kinase 3 beta (GSK-3 $\beta$ ) inhibitor (Intravenous injection, $10 \mu \mathrm{M}, 1 \mathrm{~mL}$, once a day) group $\mathrm{IV}$, and venlafaxine suspension (Oral, $0.035 \mathrm{~g} / \mathrm{kg}$, once a day) in group $\mathrm{V}$. Venlafaxine, the common used anti-depression drug in the clinic, was chose in this study. Herba Rhodiolae was purchased from Beijing Tongrentang (Beijing, China), and the dose of Herba Rhodiolae was determined through our pre-experiment and dose conversion from human to rat. Animal experiments were performed under a project license (\#2021-016) granted by the Ethics Committee of The Second Affiliated Hospital of Fujian Traditional Chinese Medical University, in compliance with the institutional guidelines for the care and use of animals.

\section{CUMS protocol}

The rats in group II-V were exposed to CUMS to induce depression, according to a modified protocol described by Dubey et al. (2015) and Lu et al. (2017) $(12,18)$. The stressors used in the present study consisted of water deprivation for $24 \mathrm{~h}$, food deprivation for $24 \mathrm{~h}$, soiled bedding for $24 \mathrm{~h}$, cage tilting $\left(45^{\circ}\right)$ for $24 \mathrm{~h}$, inversion of light-dark cycle 
for $24 \mathrm{~h}$, social isolation for $12 \mathrm{~h}$, restraint stress for $2 \mathrm{~h}$, hanging by the tail for $30 \mathrm{~min}$, and swimming in cold water $\left(4^{\circ} \mathrm{C}\right.$ ) for $5 \mathrm{~min}$. Each rat was randomly subjected to 1 stressor every day, with each stressor applied to each rat at least 1 time but less than 3 times. The CUMS stimulation was performed for 14 consecutive days.

\section{Bebavioral tests}

The fluid consumption test was performed to evaluate potential anhedonia. Prior to the test, the rats were supplied with 2 bottles of $1 \%$ sucrose solution on day 1 , and 1 bottle of $1 \%$ sucrose solution and 1 bottle of pure water on day 2. On day 3, the rats were also provided 1 bottle of sucrose solution and 1 bottle of pure water, but the position of the 2 bottles were switched every $6 \mathrm{~h}$. After 3 days of acclimatization, the rats were subjected to food and water deprivation for $12 \mathrm{~h}$, and then was given 1 bottle of sucrose solution and 1 bottle of pure water. After $12 \mathrm{~h}$, the sucrose preference was calculated as follows: sucrose preference $(\%)=$ sucrose solution consumption/(sucrose solution consumption + water consumption) $\times 100 \%$.

To evaluate locomotor activity, the open field test was conducted in a dark box with a square floor $(80 \mathrm{~cm} \times$ $80 \mathrm{~cm}$ ) and walls $45 \mathrm{~cm}$ in height. The floor was further subdivided into 16 equal squares $(20 \mathrm{~cm} \times 20 \mathrm{~cm})$ with white stripes. The rat was initially placed in the center of floor, and its behavior was recorded for $5 \mathrm{~min}$ with a camera, after environmental adaptation for $2 \mathrm{~min}$. The score of locomotor activity was estimated by the number of crossing behaviors, with 3 paws crossing the line accounting for 1 score. Each rearing accounted for 1 score. The box was cleaned using $70 \%$ ethanol in water after each test.

To evaluate susceptibility to negative mood, the forced swimming test was conducted in a cylindrical glass (diameter $14 \mathrm{~cm}$, height $20 \mathrm{~cm}$ ) filled with water at $23{ }^{\circ} \mathrm{C}$ with a depth of $10 \mathrm{~cm}$. After swimming training $(10 \mathrm{~min})$ at $24 \mathrm{~h}$ before the test, each rat was placed individually in the glass and video recorded for $5 \mathrm{~min}$. The immobility duration was calculated for each rat.

\section{Tissue sampling and brain morphology}

After behavioral tests, the rats were sacrificed with a lethal dose of pentobarbital. Hippocampi were subsequently separated from brains of 3 individuals in each group, and hippocampus samples were immediately frozen in liquid nitrogen and stored at $-80{ }^{\circ} \mathrm{C}$. For the other 3 animals in each group, brain tissues including the hippocampus regions were fixed overnight with the mixture of $2.5 \%$ glutaraldehyde and $2.5 \%$ paraformaldehyde at $4{ }^{\circ} \mathrm{C}$. The fixed samples were washed with $0.1 \mathrm{M}$ cold phosphatebuffered saline (PBS) and post-fixed with $1 \%$ osmium in $0.1 \mathrm{M}$ PBS for $1 \mathrm{~h}$ at $4^{\circ} \mathrm{C}$. The samples were dehydrated with a gradient of acetone solution (30-100\%) and the dehydrated samples were embedded in epoxy resin overnight. The prepared samples were cut into semi-thin sections (100-120 nm), stained with 1\% toluidine blue for $5 \mathrm{~min}$, and blocked with rubber. The semi-thin sections were further cut into ultra-thin sections (40-60 nm) and stained with uranyl acetate for $30 \mathrm{~min}$. The ultra-thin sections were observed by JEM-1200EX transmission electron microscopy (TEM, JEOL, Tokyo, Japan).

\section{High performance liquid chromatography}

The glutamate level in the hippocampus was determined by high performance liquid chromatography (HPLC; Agilent 1260, Thermo Fisher Scientific, Waltham, MA, USA), according to the protocol described by Cui et al. (2017) (19). The hippocampus sample was added to homogenization buffer and centrifuged to obtain supernatant, which was suspended with O-phthaldialdehyde. The derivatization mixture was eluted in an Easy C18 analytical column (Agilent, $150 \mathrm{~mm} \times 75 \mu \mathrm{m}, 3 \mu \mathrm{m}$ particle size), using solvent A (50 mmol/L sodium acetate buffer) and solvent $\mathrm{B}$ (methanol). The gradient elution was run at a flow rate of $0.1 \mathrm{~mL} / \mathrm{min}$, during which solvent $\mathrm{B}$ concentration was initially at $30 \%$ for $5 \mathrm{~min}$, increased to $37 \%$ for $15 \mathrm{~min}$, and returned to $30 \%$ for $20 \mathrm{~min}$. The column temperature was maintained at $35{ }^{\circ} \mathrm{C}$. The glutamate was detected using a fluorescence detector, of which the excitation and emission wavelengths were set at 340 and $440 \mathrm{~nm}$, respectively.

\section{Western blot}

The proteins were isolated from hippocampus samples using radio-immunoprecipitation assay (RIPA) lysis buffer, including a cocktail of protease, phosphatase inhibitors, and phenylmethylsulfonyl fluoride (PMSF; Beyotime, Shanghai, China). The isolated proteins were separated on a $10 \%$ sodium dodecyl-sulfate polyacrylamide gel electrophoresis (SDS-PAGE) gel and transferred to polyvinylidene difluoride (PVDF) membranes (Bio- 

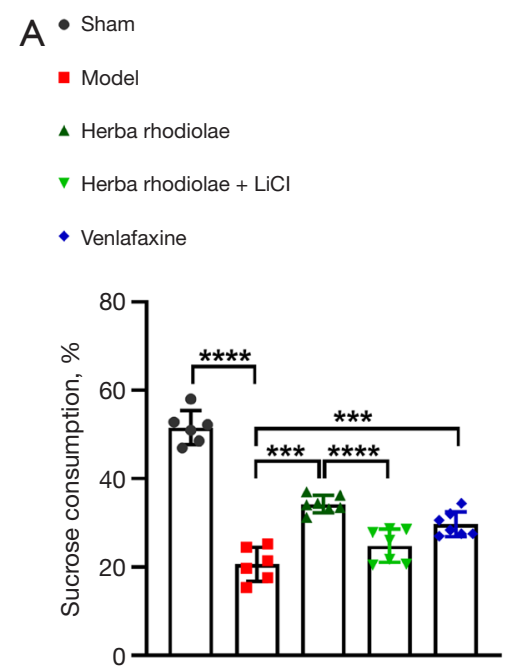

B • Sham

- Model

- Herba rhodiolae

$\checkmark$ Herba rhodiolae $+\mathrm{LiCl}$

- Venlafaxine

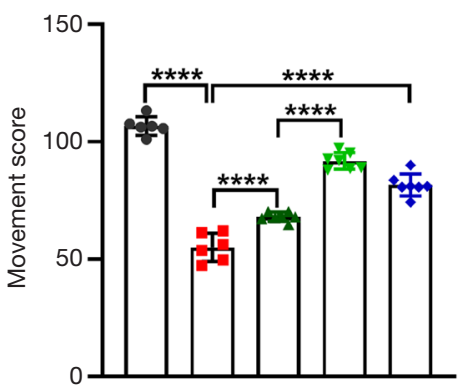

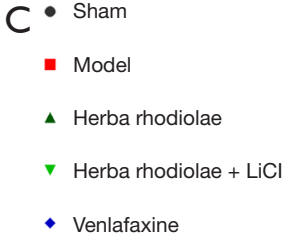

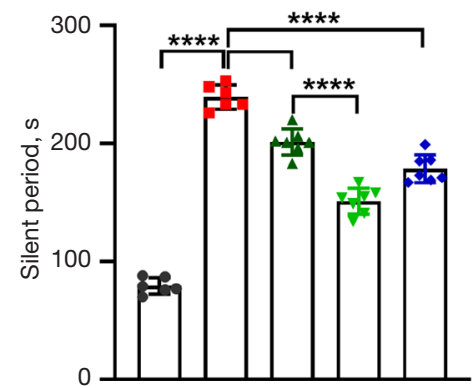

Figure 1 Effects of treatments of Herba Rhodiolae, Herba Rhodiolae plus GSK-3 $\beta$ inhibitor, and venlafaxine on depressive-like behaviors of CUMS rats. The depressive-like behaviors were evaluated with sucrose consumption using a sucrose preference test (A), locomotor activity in open field test (B), and immobility time in forced swimming test $(\mathrm{C})$. ${ }^{*} \mathrm{P}<0.05,{ }^{* *} \mathrm{P}<0.01$, ${ }^{* *} \mathrm{P}<0.001$, and ${ }^{* * * *} \mathrm{P}<0.0001$. CUMS, chronic unpredictable mild stress; GSK-3 $\beta$, glycogen synthase kinase 3 beta.

Rad, Hercules, CA, USA). After blocking with $5 \%$ nonfat milk in Tris-buffered saline containing $0.1 \%$ Tween 20 (TBST), the membrane was incubated overnight at $4{ }^{\circ} \mathrm{C}$ with monoclonal rabbit primary antibodies specific to tropomyosin receptor kinase B (TrkB), brain derived neurotrophic factor (BDNF), P-GSK3- $\beta$, GSK3- $\beta$, LC3I, LC3II, Beclin 1, and glyceraldehyde 3-phosphate dehydrogenase (GAPDH; acting as internal control). After washing with TBST, the membrane was incubated for $1 \mathrm{~h}$ at room temperature with horseradish peroxidase (HRP)-conjugated secondary antibodies. The protein band was visualized using the Immun-StarTM HRP Chemiluminescence Kit (Bio-Rad) with ImageQuant LAS 4000 system (GE Healthcare, Hino, Japan). The intensities of protein bands were estimated using Image $\mathrm{J}$ analysis software (https://imagej.nih.gov/ij/).

\section{Statistical analysis}

One-way analysis of variance (ANOVA) test was applied for comparison among groups, in combination with Turkey's post-hoc test for multiple comparisons. Prior to one-way ANOVA, the normality of data was verified by the Shapiro-Wilk test. All statistical analyses were performed on Graph Pad Prism 5.0 (Graph Pad Software, San Diego, CA, USA). A P value $<0.05$ indicated a significant difference.

\section{Results}

\section{Behavioral performance}

In the sucrose preference test, the percentage of sucrose consumption in group I $(51.59 \% \pm 3.85 \%)$ was significantly greater than that in the other 4 groups $\left[F_{(4,28)}=80.92, \mathrm{P}<0.05\right]$. The sucrose consumption of group III $(29.68 \% \pm 3.85 \%)$ was significantly greater than that in group IV $(24.87 \% \pm 3.76 \%)$ and group II $(29.68 \% \pm 3.85 \%)$. The percentage of sucrose consumption in group II was significantly smaller than that in group $\mathrm{V}(29.68 \% \pm 2.80 \%)$ (Figure $1 A)$. In the open field test, significant differences were observed in all comparisons between groups $\left[F_{(4,28)}=144.50, \mathrm{P}<0.05\right]$. The average movement score was 106.80 (SD 3.95) in group I, 91.86 (SD 3.54) in group IV, 81.63 (SD 4.67) in group V, 68.09 (SD 1.92) in group III, and 55.05 (SD 5.99) in group II (Figure 1B). In the forced swimming test, significant differences were observed in all comparisons between different groups $\left[F_{(4,28)}=201.10, \mathrm{P}<0.05\right]$. The average of immobility time was 239.30 seconds (SD 10.33) in group II, 201.30 (SD 11.07) in group III, 178.60 (SD 11.75) in group V, 151.10 (SD 10.98) in group IV, and 79.50 (SD 6.89) in group I (Figure 1C).

\section{Protein expression}

The expression level of TrkB was significantly decreased in group II-V $(0.53 \pm 0.03$ for group II, $0.66 \pm 0.06$ for group 

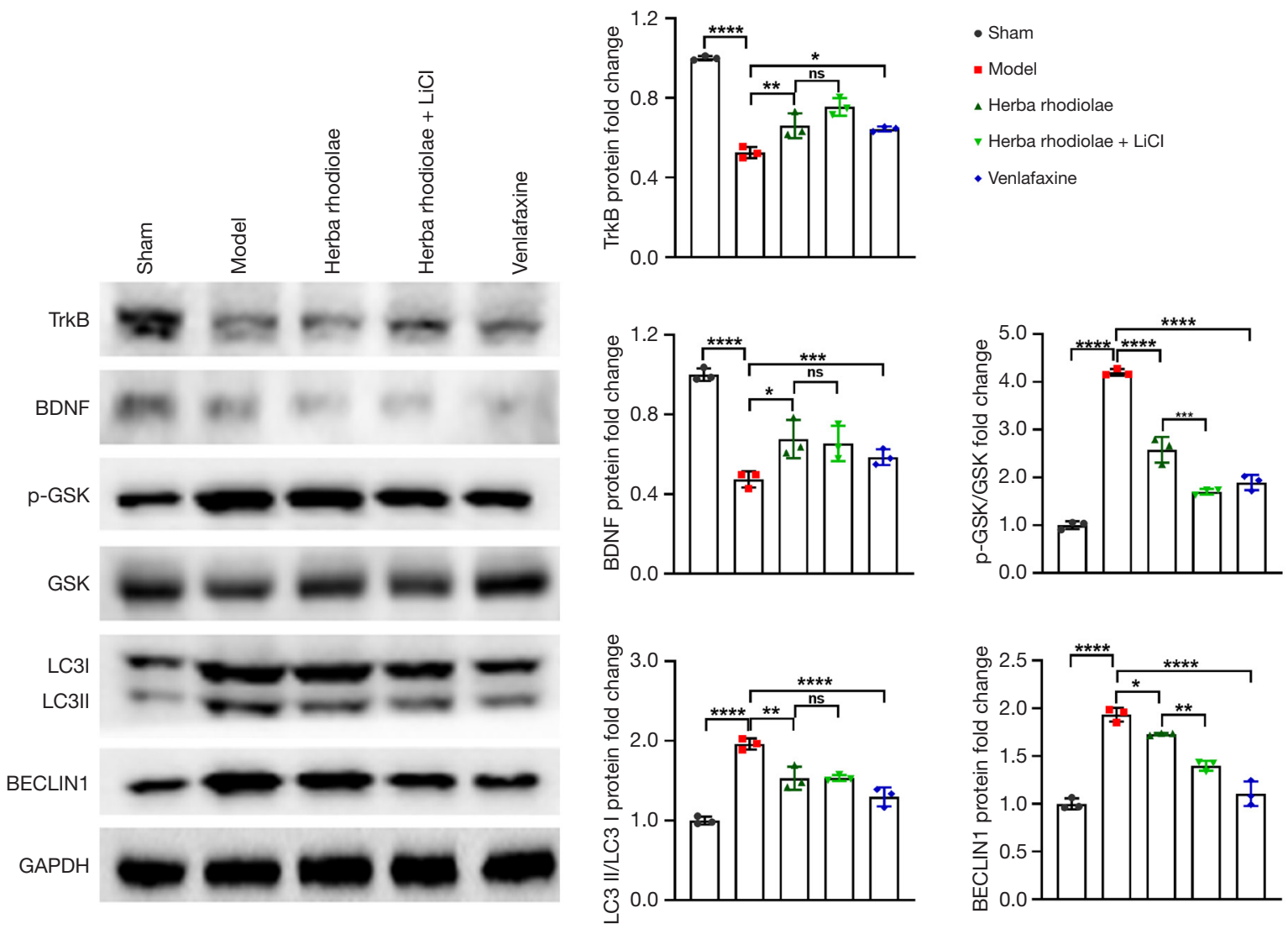

Figure 2 Effects of Herba Rhodiolae, Herba Rhodiolae plus GSK-3 $\beta$ inhibitor, and venlafaxine on protein expression in hippocampus regions of CUMS rats. The expression level of TrkB, BDNF, P-GSK3- $\beta$, GSK3- $\beta$, LC3I, LC3II, Beclin 1, and GAPDH in hippocampus was estimated, based on the intensities of protein bands (in left). ${ }^{*} \mathrm{P}<0.05,{ }^{* *} \mathrm{P}<0.01,{ }^{* * *} \mathrm{P}<0.001$, and ${ }^{* * * *} \mathrm{P}<0.0001$. CUMS, chronic unpredictable mild stress; TrkB, tropomyosin receptor kinase B; BDNF, brain derived neurotrophic factor; GAPDH, glyceraldehyde 3-phosphate dehydrogenase; GSK-3 $\beta$, glycogen synthase kinase 3 beta.

III, $0.76 \pm 0.04$ for group IV, and $0.64 \pm 0.01$ for group V),

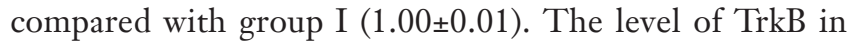
group II was significantly lower than that in group III-V $\left(F_{(4,10)}=68.70, \mathrm{P}<0.05\right)$. Significant differences were observed between group IV and group V (Figure 2). The expression of BDNF was significantly decreased in group II-V $(0.47 \pm 0.04$ for group II, $0.68 \pm 0.10$ for group III, $0.65 \pm 0.09$ for group IV, and $0.59 \pm 0.04$ for group V), compared with group I $(1.00 \pm 0.03)$. The level of BDNF was significantly lower compared with group III and IV $\left[F_{(4,10)}=27.16\right.$, $\mathrm{P}<0.05$, Figure 2]. The ratio of LC3II and LC3I was significantly increased in group II-V $(1.96 \pm 0.07$ for group II, $1.53 \pm 0.15$ for group III, $1.54 \pm 0.04$ for group IV, and $1.23 \pm 0.12$ for group $V)$, compared with group I $(1.00 \pm 0.05)$. The expression ratio of LC3II and LC3I in group II was significantly higher than that of group III-V $\left[F_{(4,10)}=43.13\right.$,
$\mathrm{P}<0.05$, Figure 2]. The expression level of $\mathrm{P}-\mathrm{GSK} / \mathrm{GSK}$ was significantly increased in group II-V $(4.20 \pm 0.07$ for group II, $2.58 \pm 0.27$ for group III, $1.70 \pm 0.06$ for group IV, and $1.89 \pm 0.16$ for group V), compared with group I $(1.00 \pm 0.08)$. Significant differences were observed in Beclin 1 expression between groups $(1.00 \pm 0.06$ for group I, $1.93 \pm 0.07$ for group II, $1.73 \pm 0.01$ for group III, and $1.40 \pm 0.05$ for group IV), except for group I and group V $(1.11 \pm 0.13)\left[F_{(4,10)}=85.13\right.$, $\mathrm{P}<0.05$, Figure 2].

\section{TEM and glutamate concentration}

The glutamate concentration in group I $(589.7 \pm 41.79)$ was significantly lower than that in group II-V $(848.00 \pm 29.31$ for group II, $750.30 \pm 5.69$ for group III, $678.70 \pm 22.50$ for group IV, and $713.30 \pm 8.51$ for group V) (Figure 3). The 
glutamate concentration in group II was significantly greater than that in group III-V. The glutamate concentration in group III was significantly higher than that in group IV. No differences were observed in comparisons between group III and group $\mathrm{V}$, group IV and group $\mathrm{V}$ $\left[F_{(4,10)}=41.94, \mathrm{P}<0.05\right]$ (Figure 3). No auto-phagosome was observed in brain neurons of rats in the group I and group IV. Significant increases of auto-phagosomes number in brain neurons, especially in the hippocampus region of animals were observed in group II compared with group III

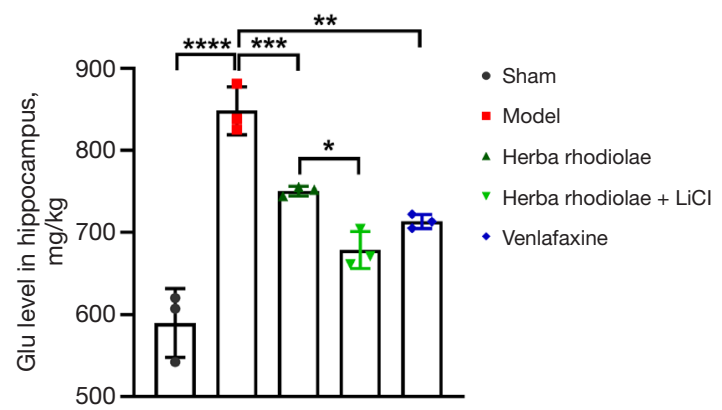

Figure 3 Effects of Herba Rhodiolae, Herba Rhodiolae plus GSK$3 \beta$ inhibitor, and venlafaxine on glutamate level in hippocampus of CUMS rats. ${ }^{*} \mathrm{P}<0.05,{ }^{* *} \mathrm{P}<0.01,{ }^{* * *} \mathrm{P}<0.001$, and ${ }^{* * * *} \mathrm{P}<0.0001$. CUMS, chronic unpredictable mild stress; GSK-3 $\beta$, glycogen synthase kinase 3 beta. and V. A larger number of auto-phagosomes were found in group V than in group III (Figure 4).

\section{Discussion}

Compared with the sham group, the sucrose consumption and locomotor activity of CUMS rats were significantly decreased, and the immobility time was contrarily increased, in agreement with previous findings (20). The decrease of sucrose consumption and locomotor activity, anhedonia, loss of interesting, and despair, respectively, are the core symptoms of human depression $(21,22)$. The depressivelike behaviors were probably related to the impairments of the central nervous system in CUMS rats, given the abnormalities in brain morphology. In morphology, a large number of auto-phagosomes were induced by CUMS stimulation in neurons, especially in the hippocampus regions. Auto-phagosome is the unique organelle involving in autophagy, which is the intracellular degradation system transporting cytoplasmic constituents to the lysosome. The autophagy activated in the brain was in consistent with the increased levels of the autophagy-related proteins Beclin-1 and LC3II/I.

Abnormality of the central nervous system was observed in physiological examinations. Specifically, glutamate level in the brain was elevated in CUMS rats compared to the sham group, which was in agreement with findings in humans
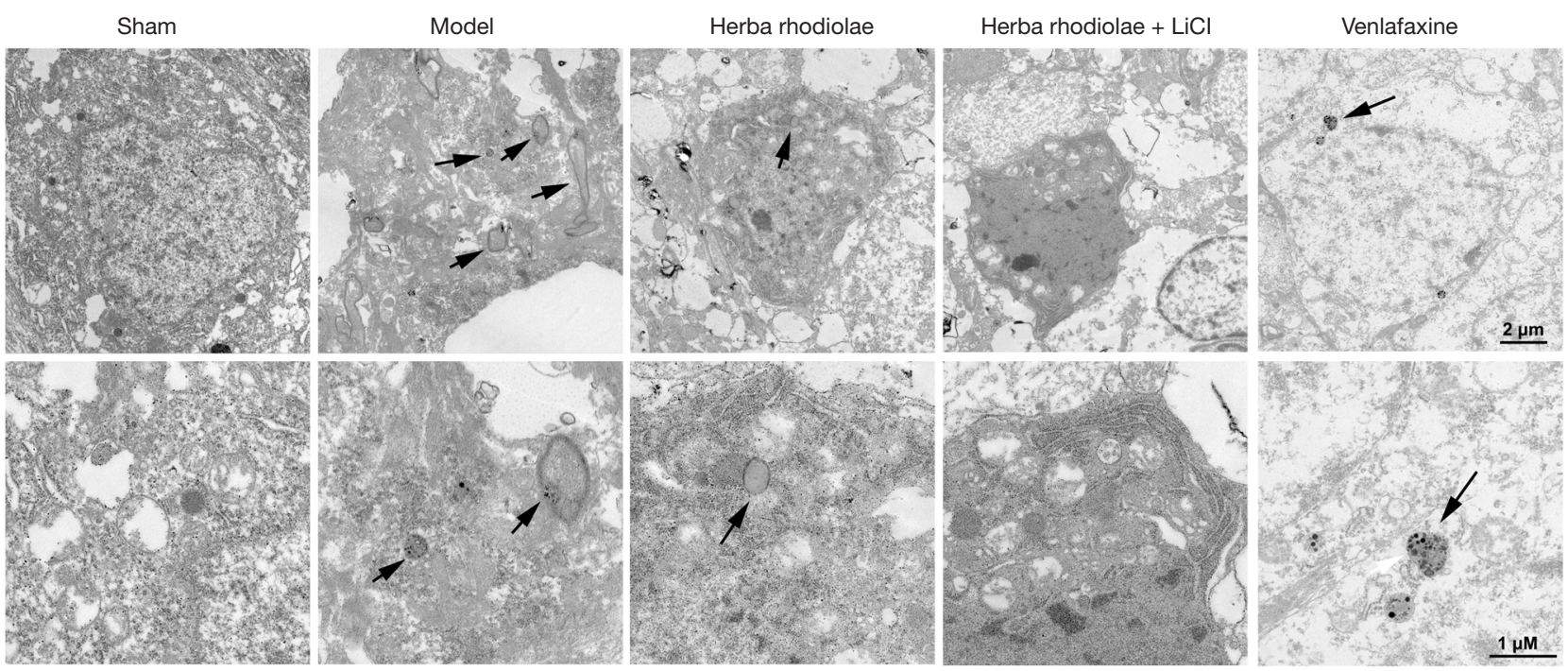

Figure 4 Effects of Herba Rhodiolae, Herba Rhodiolae plus GSK-3 $\beta$ inhibitor, and venlafaxine on the number of autophagosomes in brain of CUMS rats. The autophagosomes were indicated by arrows in the figure. CUMS, chronic unpredictable mild stress; GSK-3 $\beta$, glycogen synthase kinase 3 beta. 
(23). Glutamate is a major excitatory neurotransmitter in the central nervous system, and it is involved in synaptic plasticity and synaptogenesis (24). Glutamate released from presynaptic neurons could bind to glutamate receptors of postsynaptic neurons. Glutamate receptors mainly include $\alpha$-amino-3-hydroxy-5-methyl-4-isoxazole propionic acid (AMPA) receptor, N-methyl-D-aspartate (NMDA) receptor, and metabotropic glutamate receptors (mGluRs). The excessive glutamate likely resulted in neurotoxic-like effects in the central nervous system (25). Intracellular BDNF/TrkB signaling, which closely interacts with the glutamate system in neurons, has been demonstrated to be involved in pathological mechanism of depression. The $\mathrm{BDNF} /$ TrkB signaling has been shown to activate the PI3K/ mTOR and Ras/ERK pathways and play an important role in the maintenance and survival of neurons and synaptic plasticity (26,27). In agreement with previous findings (28), the levels of BDNF and TrkB were significantly reduced by CUMS stimulation in the present study, which were associated with the development of depression. In concert with the BDNF/TrkB signaling $(28,29)$, the expression of GSK-3 $\beta$, a component of PI3K/mTOR and Ras/ERK pathways, was contrarily increased in CUMS rats.

The depressive-like behaviors induced by CUMS stimulation were alleviated by the treatments of Herba Rhodiolae, combination of Herba Rbodiolae and GSK-3 $\beta$ inhibitor, and venlafaxine. Among these treatments, the combination of Herba Rhodiolae and GSK-3 $\beta$ inhibitor appeared to be most effective, incorporating the benefits of both Herba Rhodiolae and GSK-3 $\beta$ inhibitor. The discrepancy in treatment effect likely resulted from different effects on pathological processes. Specifically, Herba Rbodiolae, GSK-3 $\beta$ inhibitor, and venlafaxine were understood to affect the auto-phagosome number and glutamate level stimulated by CUMS in the central nervous system. Herba Rhodiolae was more effective in rehabilitating $\mathrm{BDNF} / \mathrm{TrkB}$ signaling than venlafaxine. The rehabilitation of BDNF/TrkB signaling was not observed after treatment with GSK-3 $\beta$ inhibitor. Nonetheless, GSK-3 $\beta$ inhibitor was the most effective in down-regulation of GSK-3 $\beta$ expression compared to Herba Rbodiolae and venlafaxine. These findings indicated that the combined treatments of Herba Rbodiolae and GSK-3 $\beta$ inhibitor rehabilitate the $\mathrm{BDNF} / \mathrm{TrkB}-\mathrm{GSK}-3 \beta$ signaling pathway.

\section{Acknowledgments}

Funding: This study was supported by the Fujian Provincial
Health Commission (2019-ZQN-78) and Natural Science Foundation of the Fujian Province (2020J01247)

\section{Footnote}

Reporting Checklist: The authors have completed the ARRIVE reporting checklist. Available at https://dx.doi. org/10.21037/atm-21-5849

Data Sharing Statement: Available at https://dx.doi. org/10.21037/atm-21-5849

Conflicts of Interest: All authors have completed the ICMJE uniform disclosure form (available at https://dx.doi. org/10.21037/atm-21-5849). The authors have no conflicts of interest to declare.

Ethical Statement: The authors are accountable for all aspects of the work in ensuring that questions related to the accuracy or integrity of any part of the work are appropriately investigated and resolved. Animal experiments were performed under a project license (\#2021-016) granted by the Ethics Committee of The Second Affiliated Hospital of Fujian Traditional Chinese Medical University, in compliance with the institutional guidelines for the care and use of animals.

Open Access Statement: This is an Open Access article distributed in accordance with the Creative Commons Attribution-NonCommercial-NoDerivs 4.0 International License (CC BY-NC-ND 4.0), which permits the noncommercial replication and distribution of the article with the strict proviso that no changes or edits are made and the original work is properly cited (including links to both the formal publication through the relevant DOI and the license). See: https://creativecommons.org/licenses/by-nc-nd/4.0/.

\section{References}

1. Nestler EJ, Barrot M, DiLeone RJ, et al. Neurobiology of depression. Neuron 2002;34:13-25.

2. Kessler RC, Berglund P, Demler O, et al. Lifetime prevalence and age-of-onset distributions of DSM-IV disorders in the National Comorbidity Survey Replication. Arch Gen Psychiatry 2005;62:593-602.

3. Hawton K, Casañas I Comabella C, Haw C, et al. Risk factors for suicide in individuals with depression: a systematic review. J Affect Disord 2013;147:17-28. 
4. Chopra K, Kumar B, Kuhad A. Pathobiological targets of depression. Expert Opin Ther Targets 2011;15:379-400.

5. Kallies G, Rapp MA, Fydrich T, et al. Serum brainderived neurotrophic factor (BDNF) at rest and after acute aerobic exercise in major depressive disorder. Psychoneuroendocrinology 2019;102:212-5.

6. Amidfar M, Kim YK, Wiborg O. Effectiveness of memantine on depression-like behavior, memory deficits and brain mRNA levels of BDNF and TrkB in rats subjected to repeated unpredictable stress. Pharmacol Rep 2018;70:600-6.

7. Chiu CH, Chyau CC, Chen CC, et al. Erinacine A-Enriched Hericium erinaceus Mycelium Produces Antidepressant-Like Effects through Modulating BDNF/ PI3K/Akt/GSK-3beta Signaling in Mice. Int J Mol Sci 2018;19:341.

8. Barbui C, Butler R, Cipriani A, et al. Depression in adults: drug and physical treatments. BMJ Clin Evid 2007.

9. Cuijpers P, Karyotaki E. The effects of psychological treatment of perinatal depression: an overview. Arch Womens Ment Health 2021;24:801-6.

10. Holtzheimer PE 3rd, Nemeroff CB. Advances in the treatment of depression. NeuroRx 2006;3:42-56.

11. Little A. Treatment-resistant depression. Am Fam Physician 2009;80:167-72.

12. Dubey VK, Ansari F, Vohora D, et al. Possible involvement of corticosterone and serotonin in antidepressant and antianxiety effects of chromium picolinate in chronic unpredictable mild stress induced depression and anxiety in rats. J Trace Elem Med Biol 2015;29:222-6.

13. Pan YJ, Cheng IC, Yeh LL, et al. Utilization of traditional Chinese medicine in patients treated for depression: a population-based study in Taiwan. Complement Ther Med 2013;21:215-23.

14. Li C, Huang J, Cheng YC, et al. Traditional Chinese Medicine in Depression Treatment: From Molecules to Systems. Front Pharmacol 2020;11:586.

15. Gao L, Wu C, Liao Y, et al. Antidepressants effects of Rhodiola capsule combined with sertraline for major depressive disorder: A randomized double-blind placebocontrolled clinical trial. J Affect Disord 2020;265:99-103.

16. Ming DS, Hillhouse BJ, Guns ES, et al. Bioactive compounds from Rhodiola rosea (Crassulaceae). Phytother Res 2005;19:740-3.

17. Kamel Ismail ZM, Morcos MA, Eldin Mohammad MD, et al. Enhancement of Neural Stem Cells after Induction of Depression in Male Albino Rats (A histological \& Immunohistochemical Study). Int J Stem Cells 2014;7:70-8.

18. Lu J, Shao RH, Jin SY, et al. Acupuncture ameliorates inflammatory re-sponse in a chronic unpredictable stress rat model of depression. Brain Res Bull 2017;128:106-12.

19. Cui T, Qiu HM, Huang D, et al. Abnormal levels of seven amino neuro-transmitters in depressed rat brain and determination by HPLC-FLD. Biomed Chromatogr 2017;31:e3937.

20. Wang Q, Timberlake MA 2nd, Prall K, et al. The recent progress in animal models of depression. Prog Neuropsychopharmacol Biol Psychiatry 2017;77:99-109.

21. Bai M, Zhu X, Zhang Y, et al. Abnormal hippocampal BDNF and miR-16 expression is associated with depression-like behaviors induced by stress during early life. PLoS One 2012;7:e46921.

22. Anisman H, Matheson K. Stress, depression, and anhedonia: caveats concerning animal models. Neurosci Biobehav Rev 2005;29:525-46.

23. Hashimoto K. Role of the mTOR signaling pathway in the rapid antidepressant action of ketamine. Expert Rev Neurother 2011;11:33-6.

24. Hashimoto K. Emerging role of glutamate in the pathophysiology of major depressive disorder. Brain Res Rev 2009;61:105-23.

25. Murrough JW, Abdallah CG, Mathew SJ. Targeting glutamate signalling in depression: progress and prospects. Nat Rev Drug Discov 2017;16:472-86.

26. Dwivedi Y. Brain-derived neurotrophic factor: role in depression and suicide. Neuropsychiatr Dis Treat 2009;5:433-49.

27. Leal G, Afonso PM, Salazar IL, et al. Regulation of hippocampal synaptic plasticity by BDNF. Brain Res 2015;1621:82-101.

28. Zhang SS, Tian YH, Jin SJ, et al. Isoflurane produces antidepressant effects inducing BDNF-TrkB signaling in CUMS mice. Psychopharmacology (Berl) 2019;236:3301-15.

29. Duda P, Hajka D, Wojcicka O, et al. GSK3beta: A Master Player in Depressive Disorder Pathogenesis and Treatment Responsiveness. Cells 2020;9:727.

Cite this article as: Gao L, Wu C, Liao Y, Zhang S, Zhao J. Herba Rhodiolae alleviates depression via the BDNF/TrkBGSK-3 $\beta$ signaling pathway. Ann Transl Med 2021;9(24):1758. doi: $10.21037 / \mathrm{atm}-21-5849$ 